PROCEEDINGS OF THE

AMERICAN MATHEMATICAL SOCIETY

Volume 140, Number 3, March 2012, Pages 1053-1068

S 0002-9939(2011)10941-9

Article electronically published on July 1, 2011

\title{
VOLUME MAXIMIZATION AND THE EXTENDED HYPERBOLIC SPACE
}

\author{
FENG LUO AND JEAN-MARC SCHLENKER
}

(Communicated by Jianguo Cao)

\begin{abstract}
We consider a volume maximization program to construct hyperbolic structures on triangulated 3-manifolds, for which previous progress has lead to consider angle assignments which do not correspond to a hyperbolic metric on each simplex. We show that critical points of the generalized volume are associated to geometric structures modeled on the extended hyperbolic space - the natural extension of hyperbolic space by the de Sitter space - except for the degenerate case where all simplices are Euclidean in a generalized sense.

Those extended hyperbolic structures can realize geometrically a decomposition of the manifold as the connected sum of components admitting a complete hyperbolic metric, along embedded spheres (or projective planes) which are totally geodesic space-like surfaces in the de Sitter part of the extended hyperbolic structure.
\end{abstract}

\section{INTRODUCTION}

1.1. The Casson-Rivin program and its extension. There are several ways to construct hyperbolic metrics on an ideally triangulated 3-manifold with torus boundary. The most prominent ones are Thurston's algebraic gluing equations and Casson-Rivin's angle structure. In the angle structure approach, one first introduces the notion of an angle on the triangulation and uses the Lobachevsky function to define the volume of a tetrahedron with angle assignments. In Luo07, CassonRivin's angle structure program is extended to closed triangulated 3-manifolds. The volume of an angle structure is defined and the critical points of the volume are investigated in Luo07.

The goal of this paper is to investigate the relationship between the critical points of the volume and a natural extension of the hyperbolic space by the de Sitter space. It turns out that many of the critical points have geometric meaning in terms of geometric structures based on this extended hyperbolic space.

Received by the editors June 22, 2010 and, in revised form, November 27, 2010 and December 8, 2010 .

2010 Mathematics Subject Classification. Primary 57M50; Secondary 51M10, 52B70, 57Q15.

The first author was partially supported by NSF-DMS0604352.

The second author was partially supported by the ANR program Repsurf: ANR-06-BLAN0311

(C)2011 American Mathematical Society Reverts to public domain 28 years from publication 


\subsection{Volume maximization on triangulated 3-manifolds.}

Definition 1.1. Let $M$ be a closed 3-manifold, and let $T$ be a triangulation of $M$. Recall that the triangulation $T$ is obtained as follows. Take a finite set of disjoint Euclidean tetrahedra $s_{1}, \ldots, s_{N}$ and identify all their codimension-1 faces in pairs by affine homeomorphisms. The quotient space $M$ inherits a natural triangulation, denoted by $T$. A wedge of $T$ is a couple $(e, s)$, where $s$ is a simplex of $T$ and $e$ is an edge of $s$ in the unidentified space $\bigcup_{i=1}^{N} s_{i}$. The set of wedges of $T$ will be denoted by $W(T)$, while the set of vertices, edges, 2-faces and 3-simplices of $T$ will be denoted by $V(T), E(T), F(T)$ and $S(T)$ respectively.

The main ingredient in Casson-Rivin's angle structure is based on the observation that the vertex link of an ideal hyperbolic tetrahedron is a Euclidean triangle. In our extension, we observe that the vertex link of a compact tetrahedron in the hyperbolic, spherical or Euclidean 3-space is a spherical triangle. This prompts us to propose the following definition.

Definition 1.2. An angle structure on $T$ is a function $\theta: W(T) \rightarrow(0, \pi)$ such that:

- for each edge $e$ of $T$, the sum of the $\theta(w)$ over the wedges of the form $w=(e, s), s \in S(T)$, is equal to $2 \pi$,

- for each $s \in S(T)$ and each vertex $v$ of $s, \theta\left(e_{1}, s\right)+\theta\left(e_{2}, s\right)+\theta\left(e_{3}, s\right)>\pi$, where the $e_{i}$ are the three edges of $s$ adjacent to $v$,

- for each $s \in S(T)$ and each vertex $v$ of $s, \theta\left(e_{1}, s\right)+\theta\left(e_{2}, s\right)<\theta\left(e_{3}, s\right)+\pi$, and similarly for permutations of $1,2,3$.

We denote by $A S(T)$ the space of angle assignments on $T$.

The geometric meaning of the first condition is that, if the geometric structures on the simplices can be glued along the faces (this is discussed below), then the angles add up at the edges so that there is no singularity there. The second and the third conditions mean that for each simplex the link of each vertex is a spherical triangle.

It is quite possible that, in some cases, $A S(T)$ might be empty. However, all manifolds do admit a triangulation for which $A S(T)$ is non-empty $[\mathrm{KL}$.

There is a well-defined manner, explained in [Luo07] and recalled in sections 4-5, for associating to an angle assignment $\theta \in A S(T)$ a number which is, in a precise way, a generalized volume. This defines a function $V: A S(T) \rightarrow \mathbb{R}$. This "volume" is defined in terms of a natural extension of the Schläfli formula, so that it automatically satisfies this identity.

If $\theta_{0}$ is a critical point of $V$ in $A S(T)$ such that all the angles assigned to all simplices of $T$ are the dihedral angles of a hyperbolic simplex, then, thanks to the Schläfli formula, the lengths of an edge in the wedges containing it match, so that the faces of the simplices can be glued isometrically and $\theta_{0}$ defines a hyperbolic metric on $M$. One of the main points of this paper is that an extension of this statement holds when $\theta_{0}$ does not assign to all simplices of $T$ the dihedral angles of a hyperbolic simplex.

1.3. Extended hyperbolic structures. There is a rather natural extension of the hyperbolic space by the de Sitter space, used for instance in [Sch98, Sch01] in a polyhedral context somewhat reminiscent of the arguments followed here. We call $H S^{3}$ this extended hyperbolic space, so that $H S^{3}$ contains an open subset 
isometric to the 3-dimensional hyperbolic space and another open subset isometric to the quotient of the de Sitter space by the antipodal map.

Given a 3 -dimensional manifold $M$, an $H S$-structure on $M$ is a geometric structure locally modelled on $H S^{3}$. This is explained in section 2 .

1.4. The main result. The main result presented here is that most critical points of the volume $V$ on the interior of $A S(T)$ have a natural interpretation in terms of HS-structures.

Theorem 1.3. Let $\theta \in A S(T)$ be a critical point of $V$. Then one of the following applies:

(1) $\theta$ corresponds to a spherical metric on $M$ (for each simplex of $T$, the angles are the angles of a spherical simplex, and those simplices can be glued isometrically along their faces, yielding a spherical metric).

(2) $\theta$ corresponds to an HS-structure on $M$. If this extended hyperbolic structure has a de Sitter part, then it contains a totally geodesic surface homeomorphic to $S^{2}$ or $\mathbb{R} P^{2}$, which is a normal surface in $T$.

(3) All simplices in $T$ are either Euclidean or flipped Euclidean (see below). The volume $V(\theta)$ is then a non-negative integer multiple of $2 \pi^{2}$. If at least one simplex is flipped, then $V>0$ and $(M, T)$ contains a normal surface homeomorphic to a sphere or a projective plane.

The first case should be clear and can happen only if $M$ is finitely covered by the 3 -sphere. In the second case, the totally geodesic space-like surfaces in the de Sitter parts of the HS-structure realize geometrically a decomposition of $M$ into irreductible parts, each of which carries a hyperbolic metric. The third case is quite special, and presumably it can occur only in very specific cases; see below.

1.5. Geometric interpretation. The first idea in Theorem 1.3 is that considering HS-structures radically simplifies the way in which one can find hyperbolic structures on 3-manifolds by a critical point argument. Indeed, if $M$ is reducible, there might still be a critical point of $V$ on $A S(T)$, corresponding not to a hyperbolic metric (which is impossible if $M$ is reducible) but to an HS-structure, with a de Sitter part corresponding to each incompressible sphere in $M$.

There is however a limit to this argument, as it stands. Given an HS-structure $h$ on $M$, the set of its hyperbolic points is a domain $N \subset M$ on which the restriction of $h$ defines a complete hyperbolic metric. The de Sitter parts of $h$ are topologically either products of $S^{2} \times \mathbb{R}$ or products of a projective plane by an interval. This means that each connected component of the boundary of $N$ has to be either a sphere or a projective plane, which is a very restrictive condition.

1.6. Further possible extensions. The construction of HS-structures associated to critical points of $V$ on $A S(T)$ suggests that a further extension of the space of angle assignments $A S(T)$ should be possible, allowing for instance for angle assignments such that the sum of angles at a vertex of a simplex is equal to or less than $\pi$. Such angle assignments would correspond geometrically (at critical points of $V$ ) to triangulations with at least one vertex in the de Sitter part of the HS-structure obtained. This line of investigation is not pursued here. 


\section{EXTENDED HYPERBOLIC STRUCTURES}

2.1. The extended hyperbolic space. One way to define this extension is to consider a strictly convex quadric $Q$ in the projective 3 -space $\mathbb{R} P^{3}$. Given two distinct points $x, y \in \mathbb{R} P^{3} \backslash Q$, let $D$ be the projective line containing $x$ and $y$. If $D$ intersects $Q$, let $a, b$ be the points of intersection between $D$ and $Q$, chosen so that $a, x, y, b$ occur in this cyclic order on $D$. Then define the Hilbert distance between $x$ and $y$ as

$$
d_{H}(x, y)=\frac{1}{2} \log [a, b ; x, y],
$$

where $[\cdot, \cdot ; \cdot, \cdot]$ denotes the cross-ratio. If $D$ does not intersect $Q$, use the same formula with $a, b$ replaced by the complex intersections of the line containing $x, y$ with $Q$. This defines a "distance" in which the ball bounded by $Q$ can be interpreted as a projective model of $H^{3}$, while the outside is a projective model of the quotient of the de Sitter space $d S^{3}$ by the antipodal map. In particular, $d_{H}(x, y)$ can be:

- Real and negative if $x, y$ are in the ball bounded by $Q$ and this defines inside $Q$ a projective model of the hyperbolic 3-dimensional space (known as the Klein model).

- Real and positive if $x, y$ are outside the ball bounded by $Q$ and the line joining them intersects $Q$ in two points. This line is then time-like in $d S^{3}$.

- In $i(0, \pi)$ if $x, y$ are outside $Q$ and the line containing them does not intersect $Q$. This line is then space-like in $d S^{3}$.

- In $i \pi / 2+\mathbb{R}$ if $x$ is inside the ball bounded by $Q$ and $y$ is outside it.

- 0 if the line joining $x$ and $y$ is tangent to $Q$. This line is then light-like in $d S^{3}$.

The same construction also works in dimension 2, yielding the extended hyperbolic plane $H S^{2}$, or in higher dimensions.

The space $H S^{3}$ can also be interpreted as a "natural" projectivization of the 4-dimensional Minkowski space $\mathbb{R}^{3,1}$. That is, $H S^{3}$ can be identified with the space of lines containing the origin in $\mathbb{R}^{3,1}$. The hyperbolic part of $H S^{3}$ corresponds to the time-like lines, the quadric $Q$ corresponds to the light-like lines, and the de Sitter part of $H S^{3}$ corresponds to the space-like lines.

2.2. The double cover. It is sometimes helpful to consider the double cover $\tilde{H} S^{3}$ of $H S^{3}$. It is diffeomorphic to $S^{3}$ and has two hyperbolic components each isometric to $H^{3}$ and one de Sitter component isometric to the full de Sitter space $d S^{3}$. The same works in any dimension. $\tilde{H} S^{3}$ is composed of two copies of the hyperbolic 3 -space and of one copy of the whole de Sitter space.

Just as $H S^{3}$ is the projectivization of $\mathbb{R}^{3,1}, \tilde{H} S^{3}$ can be considered as the space of geodesic rays with endpoint at 0 in $\mathbb{R}^{3,1}$.

2.3. The isometry group. The action of $P S L(2, \mathbb{C})$ on $H^{3}$ extends to $H S^{3}$. Indeed $P S L(2, \mathbb{C})$ is isomorphic to $S O_{0}(3,1)$, the identity component of the group of isometries fixing the origin in $\mathbb{R}^{3,1}$, and $S O_{0}(3,1)$ therefore acts on the projectivization of $\mathbb{R}^{3,1}$. Similarly $S O_{0}(3,1)$ acts on $\tilde{H} S^{3}$ considered as the space of geodesic rays starting from 0 in $\mathbb{R}^{3,1}$.

It should be noted, however, that all elements of $\operatorname{PSL}(2, \mathbb{C})$ have some fixed points in $H S^{3}$. Indeed, elements of $P S L(2, \mathbb{C})$ act by isometries on the hyperbolic component of $H S^{3}$, so that they can be classified as elliptic (with a fixed point in 
the hyperbolic part), parabolic (with a fixed point at infinity, in the quadric $Q$ ), or loxodromic (with two fixed points in $Q$ ). Both elliptic and parabolic isometries fix a plane in $H^{3}$ and a point in the de Sitter part of $H S^{3}$.

2.4. Extended hyperbolic structures. An HS-structure on a closed 3-dimensional manifold $M$ is a geometric structure locally modeled on $H S^{3}$, with transformation group $P S L(2, \mathbb{C})$.

If $h$ is an HS-structure on $M$, the set of points of $M$ where $h$ is locally hyperbolic is an open subset of $M$, which we denote by $M_{H}$, and the restriction of $h$ to $M_{H}$ is a complete hyperbolic metric. Similarly, the set of points of $M$ where $h$ is locally modeled on the de Sitter space is an open subset of $M$, for which we use the notation $M_{d S}$. Then $M_{H} \cup M_{d S}$ is dense is $M$, and its complement is a surface.

We will not discuss here the interesting questions concerning the notions of completeness which should be applied to HS-structures. Since all elements of $\operatorname{PSL}(2, \mathbb{C})$ act on $H S^{3}$ with fixed points, there is obviously no non-trivial quotient of $H S^{3}$ by a discrete group acting freely. This should be compared to the known situation for 3-dimensional de Sitter manifolds, where the only quotients of $d S^{3}$ are $d S^{3}$ itself and its quotient by the antipodal map. However much more freedom presumably happens if one considers HS-structures with singular points, in particular corresponding to points "at infinity".

\section{TRIANGles}

3.1. The cosine formula. Let us begin with a recall of the cosine law.

Lemma 3.1. Let $\alpha_{1}, \alpha_{2}, \alpha_{3}$ be the (interior) angles of a hyperbolic (or spherical or Eulidean) triangle, and let $a_{1}, a_{2}, a_{3}$ be the lengths of the opposite edges. Then for hyperbolic triangles,

$$
\cosh \left(a_{1}\right)=\frac{\cos \left(\alpha_{1}\right)+\cos \left(\alpha_{2}\right) \cos \left(\alpha_{3}\right)}{\sin \left(\alpha_{2}\right) \sin \left(\alpha_{3}\right)}
$$

for spherical triangles,

$$
\cos \left(a_{1}\right)=\frac{\cos \left(\alpha_{1}\right)+\cos \left(\alpha_{2}\right) \cos \left(\alpha_{3}\right)}{\sin \left(\alpha_{2}\right) \sin \left(\alpha_{3}\right)},
$$

and for Euclidean triangles,

$$
1=\frac{\cos \left(\alpha_{1}\right)+\cos \left(\alpha_{2}\right) \cos \left(\alpha_{3}\right)}{\sin \left(\alpha_{2}\right) \sin \left(\alpha_{3}\right)} .
$$

We will unify all of these three formulas using complex numbers. To this end, in the sequel we use

Definition 3.2. $\cosh$ is the restriction of the function $x \mapsto\left(e^{x}+e^{-x}\right) / 2$ to $\mathbb{R}_{<0} \cup$ $[0, i \pi] \cup\left(i \pi+\mathbb{R}_{>0}\right)$.

With this definition, cosh is a bijection from its domain of definition to $\mathbb{R}$.

3.2. The triangle inequality. Consider now a triangle in $S^{2}$, of angles $\alpha_{1}, \alpha_{2}, \alpha_{3}$. It is a simple exercise to check that those angles satisfy the following inequalities:

$$
\begin{aligned}
& \alpha_{2}+\alpha_{3}<\alpha_{1}+\pi, \\
& \alpha_{1}+\alpha_{3}<\alpha_{2}+\pi, \\
& \alpha_{2}+\alpha_{1}<\alpha_{3}+\pi .
\end{aligned}
$$


Using the exterior angles rather than the interior angles, equation $\left(T I_{1}\right)$ can be written as

$$
\left(\pi-\alpha_{1}\right)<\left(\pi-\alpha_{2}\right)+\left(\pi-\alpha_{3}\right)
$$

which is the triangle inequality for the dual triangle in the sphere.

3.3. A classification of Möbius triangles. Following Luo93, we consider here a generalization of the notion of spherical (or hyperbolic) triangle.

Definition 3.3. A Möbius triangle is a triple $\left(\alpha_{1}, \alpha_{2}, \alpha_{3}\right) \in(0, \pi)^{3}$. Given a Möbius triangle, its Möbius edge lengths are the complex numbers $\left(a_{1}, a_{2}, a_{3}\right)$ defined by equation (3.1), with the definition of cosh given above.

First of all, all Euclidean, hyperbolic and spherical triangles are Möbius triangles. By comparing (3.1), (3.2) and (3.3), we see that for a hyperbolic triangle with edge lengths $a_{1}, a_{2}, a_{3}$, its Möbius edge lengths are $-a_{1},-a_{2},-a_{3} \in \mathbf{R}_{<0}$. For a spherical triangle of edge lengths $a_{1}, a_{2}, a_{3}$, its Möbius edge lengths are $i a_{1}, i a_{2}, i a_{3}$. The Möbius edge lengths of a Euclidean triangle are zero. We will mainly work with Möbius edge lengths below and will use the term edge length for Möbius edge length.

The rationale for the terminology used here is that, for any triple $\left(\alpha_{1}, \alpha_{2}, \alpha_{3}\right) \in$ $(0, \pi)^{3}$, there exists a triangle in the complex plane bounded by three circles, unique up to Möbius transformation, so that its inner angles are the $\alpha_{i}$ 's. The constructions used below are however based mostly on the extended hyperbolic plane and on real projective geometry, rather than complex projective geometry. However sticking to the term "Möbius triangle" should be helpful to the reader insofar as it is closer to the previous works on the subjects, e.g. [Luo07, Luo93].

Lemma 3.4. Let $T=\left(\alpha_{1}, \alpha_{2}, \alpha_{3}\right)$ be a Möbius triangle, let $s=\alpha_{1}+\alpha_{2}+\alpha_{3}$, and let $a_{i}$ be the edge lengths of $T$. Exactly one of the following five cases applies:

(1) $T$ is spherical: $s>\pi$, and the triangle inequalities $\left(T I_{1}\right),\left(T I_{2}\right),\left(T I_{3}\right)$ hold. Then $a_{1}, a_{2}, a_{3} \in i(0, \pi)$.

(2) $T$ is hyperbolic: $s<\pi$. Then the triangle inequalities $\left(T I_{1}\right),\left(T I_{2}\right),\left(T I_{3}\right)$ hold, and $a_{1}, a_{2}, a_{3}<0$.

(3) $T$ is Euclidean: $s=\pi$. Then the triangle inequalities $\left(T I_{1}\right),\left(T I_{2}\right),\left(T I_{3}\right)$ hold, and $a_{1}=a_{2}=a_{3}=0$.

(4) $T$ is flipped hyperbolic: $\alpha_{2}+\alpha_{3}>\alpha_{1}+\pi$ (or similarly after a permutation of $1,2,3)$. Then $a_{1}<0$ while $a_{2}, a_{3} \in i \pi+\mathbb{R}_{>0}$.

(5) $T$ is flipped Euclidean: $\alpha_{2}+\alpha_{3}=\alpha_{1}+\pi$ (or similarly after a permutation of $1,2,3)$. Then $a_{2}=a_{3}=i \pi$ and $a_{1}=0$.

The proof goes as follows. Given an index $i \in\{1,2,3\}$, the $i$-th flip $F_{i}$ is the involution of $(0, \pi)^{3}$ so that $F_{i}\left(\alpha_{1}, \alpha_{2}, \alpha_{3}\right)=\left(\beta_{1}, \beta_{2}, \beta_{3}\right)$ where $\beta_{i}=\alpha_{i}$ and if $j \neq i$, $\beta_{j}=\pi-\alpha_{j}$. Now given any $\left(\alpha_{1}, \alpha_{2}, \alpha_{3}\right) \in(0, \pi)^{3}$, either $s=\alpha_{1}+\alpha_{2}+\alpha_{3}<\pi$, or $s=\pi$ or $s>\pi$. In the first case $s=\pi, T$ is Euclidean and Lemma 3.4(3) holds. In the second case $s<\pi, T$ is hyperbolic and Lemma 3.4(2) holds. In the case of $s>\pi$, then either $T$ is spherical and Lemma 3.4(1) holds or there is a flip $F_{i}$ so that $F_{i}\left(\alpha_{1}, \alpha_{2}, \alpha_{3}\right)$ is a Euclidean or hyperbolic triangle. Now the result follows from Lemma 3.4(1), (2) and (3) and the following sublemma, whose proof is a direct consequence of the definition of Möbius edge length given in equation (3.1). 
Sublemma 3.5. Let $T=\left(\alpha_{1}, \alpha_{2}, \alpha_{3}\right)$ be a Möbius triangle and let $a_{1}, a_{2}, a_{3}$ be the edge lengths of $T$. Let $F_{i}\left(\alpha_{1}, \alpha_{2}, \alpha_{3}\right)$ be the $i$-th flip of $T$ so that its Möbius edge lengths are $b_{1}, b_{2}, b_{3}$. Then $b_{i}=a_{i}$ and for $j \neq i, b_{j}=\sqrt{-1} \pi-a_{j}$.

The following lemma shows that the edge lengths determine the shape of a Möbius triangle.

Lemma 3.6. Let $A=\left(\alpha_{1}, \alpha_{2}, \alpha_{3}\right)$ and $B=\left(\beta_{1}, \beta_{2}, \beta_{3}\right)$ be two Möbius triangles which are not Euclidean or flipped Euclidean. If the corresponding edge lengths of $A$ and $B$ are the same, then $\alpha_{i}=\beta_{i}$ for all $i$.

The proof follows from the cosine law that

$$
\cos \left(\alpha_{1}\right)=\frac{-\cosh \left(a_{1}\right)+\cosh \left(a_{2}\right) \cosh \left(a_{3}\right)}{\sinh \left(a_{2}\right) \sinh \left(a_{3}\right)} .
$$

This law shows that lengths determine the angles.

3.4. Geometric realization of triangles. The classification of Möbius triangles in Lemma 3.4 has a natural interpretation in terms of triangles in the extended hyperbolic plane $\tilde{H} S^{2}$. There is no interpretation needed for spherical, Euclidean or hyperbolic triangle, but flipped hyperbolic triangles correspond to triangles in $\tilde{H S}{ }^{2}$ with one vertex and two in the other.

Suppose $t$ is such a flipped hyperbolic triangle, with vertices $v_{1}, v_{2}, v_{3}$, with $v_{1}$ in one copy of $H^{2}$ and $v_{2}, v_{3}$ in the other. Let $\alpha_{i}$ be the angle of $t$ at $v_{i}, 1 \leq i \leq 3$. Those angles can be understood by "flipping" $t$, that is, considering the triangle $t^{\prime}$ with vertices $v_{1}^{\prime}, v_{2}, v_{3}$, where $v_{1}^{\prime}$ is the antipode of $v_{1}$ in $\tilde{H} S^{2}$. Here $t^{\prime}$ is a "usual" hyperbolic triangle, and its angles are $\beta_{1}=\alpha_{1}, \beta_{2}=\pi-\alpha_{2}, \beta_{3}=\pi-\alpha_{3}$. Since $t^{\prime}$ is a hyperbolic triangle, its angles satisfy

$$
\beta_{1}+\beta_{2}+\beta_{3}<\pi
$$

which translates as

$$
\alpha_{1}+\pi<\alpha_{2}+\alpha_{3}
$$

the inverse triangle inequality for $t$. Similarly, $t$ satisfies the triangle inequality

$$
\pi+\beta_{2}>\beta_{1}+\beta_{3} \text { and } \pi+\beta_{3}>\beta_{1}+\beta_{2},
$$

which translates as

$$
\alpha_{1}+\alpha_{2}<\pi+\alpha_{3} \text { and } \alpha_{1}+\alpha_{3}<\pi+\alpha_{2} .
$$

This shows that $\left(\alpha_{1}, \alpha_{2}, \alpha_{3}\right)$ is a flipped hyperbolic triangle according to Lemma 3.4 . The same argument can be used backwards, to show that any flipped hyperbolic triangle in the sense of Lemma 3.4 is the triple of angles of a flipped hyperbolic triangle in $\tilde{H} S^{2}$.

Flipped Euclidean triangles can be understood in the same way but by taking a limit. The usual Euclidean triangles can be considered as limits of hyperbolic triangles of diameter going to zero - actually, a blow-up procedure is necessary, since what we really want to consider are sequences of degenerating hyperbolic triangles for which the angles, and therefore the ratio of the edge lengths, has a limit. This can also be done for flipped hyperbolic triangles, with vertices converging either to a point in the one copy of the hyperbolic plane of $\tilde{H S}$ or to its antipode in the other copy.

Putting this together, we obtain the following statement. 
Lemma 3.7. Let $T=\left(\alpha_{1}, \alpha_{2}, \alpha_{3}\right)$ be a Möbius triangle.

(1) If $T$ is spherical, there is a triangle $t \subset S^{2}$, unique up to the action of $O(3)$, with angles $\alpha_{1}, \alpha_{2}$ and $\alpha_{3}$.

(2) If $T$ is hyperbolic, there is a triangle $t \in H^{2}$, unique up to the hyperbolic isometries, with angles $\alpha_{1}, \alpha_{2}, \alpha_{3}$.

(3) If $T$ is Euclidean, there is a triangle $t \subset \mathbb{R}^{2}$, unique up to isometries and homotheties, with angles $\alpha_{1}, \alpha_{2}, \alpha_{3}$. In other words, there is a sequence of hyperbolic triangles $\left(t_{n}\right)_{n \in \mathbb{N}}$ in $H^{2}$, with angles $\alpha_{1, n}, \alpha_{2, n}, \alpha_{3, n}$ converging to $\alpha_{1}, \alpha_{2}, \alpha_{3}$, respectively.

(4) If $T$ is flipped hyperbolic, there is a $t \in \tilde{H} S^{2}$, with one vertex in one copy of $\mathrm{H}^{2}$ and two in the other, with angles $\left(\alpha_{1}, \alpha_{2}, \alpha_{3}\right)$. It is unique up to the action of $O(2,1)$ on $\tilde{H} S^{2}$.

(5) If $T$ is flipped Euclidean, there is a sequence of flipped hyperbolic triangles $\left(t_{n}\right)_{n \in \mathbb{N}}$ in $\tilde{H} S^{2}$, with angles $\alpha_{1, n}, \alpha_{2, n}, \alpha_{3, n}$ converging to $\alpha_{1}, \alpha_{2}, \alpha_{3}$, respectively.

\section{Three-Dimensional SIMPLICES}

4.1. Angle system. We now consider 3-dimensional simplices and assign to each wedge an angle, as follows.

Definition 4.1. Suppose $s$ is a tetrahedron. Then an angle system on $s$ is a function $\theta:\{(e, s) \mid e$ is an edge of $s\} \rightarrow(0, \pi)$ so that for three edges $e_{i}, e_{j}, e_{k}$ ending at a vertex $v$ of $s$, the three angles $\theta\left(e_{i}, s\right), \theta\left(e_{j}, s\right), \theta\left(e_{k}, s\right)$ are the inner angles of a spherical triangle. Let $A S(s)$ be the space of all angle systems on $s$. An angled 3 -simplex is a 3 -simplex together with an angle system.

If $T$ is a triangulation of a closed 3-manifold $M$, an angle system on $T$ is a function $\theta$ defined on the set of all wedges $\{(e, s) \mid e$ is an edge of a tetrahedron $s\}$ so that

- for each edge $e$ of $T$, the sum of the values of $\theta$ on the wedges having $e$ as their edge is equal to $2 \pi$,

- for each 3-simplex $s$ in $T$, the restriction of $\theta$ to all wedges in $s$ forms an angle system.

In the paper [Luo07, the geometric prototype of an angled 3-simplex is the Möbius tetrahedron, namely, a topological tetrahedron in $\mathbb{R}^{3}$ bounded by four 2spheres so that the dihedral angles at edges are less than $\pi$. However, there are angled 3-simplices which cannot be realized as Möbius 3-simplices. Our main observation is that, in terms of HS-geometry, these angled 3-simplices all have a geometric meaning. Furthermore, the edge lengths, volume, and Schläfli formula can be generalized to the HS-geometry. These generalizations are exactly the underlying geometric meaning of the corresponding notions defined in Luo07.

\subsection{Face angles.}

Definition 4.2. Let $\alpha=\left(\alpha_{12}, \cdots, \alpha_{34}\right) \in A S(s)$. The face angles of $\alpha$ are the numbers $\beta_{i j k} \in(0, \pi)$ defined, for $\{i, j, k, l\}=\{1,2,3,4\}$, by the formula

$$
\cos \left(\beta_{j k}^{i}\right)=\frac{\cos \left(\alpha_{i l}\right)+\cos \left(\alpha_{i j}\right) \cos \left(\alpha_{i k}\right)}{\sin \left(\alpha_{i j}\right) \sin \left(\alpha_{i k}\right)} .
$$


The geometric meaning of the face angle is as follows. According to the definition, at the $i$-th vertex $v_{i}$, the angles $\alpha_{i j}, \alpha_{i k}, \alpha_{i l}$ are the inner angles of a spherical triangle $A_{j k l}$, which can be considered as the link $v_{i}$ in the tetrahedron. Then the face angle $\beta_{j k}^{i}$ is the $j k$-th edge length of $A_{j k l}$. By definition, face angles are then in $(0, \pi)$.

4.3. Edge lengths. Using the face angles, we make each codimension-1 face of an angled tetrahedron $s$ a Möbius triangle. Thus, by Definition 3.2, we can define for each edge in each face an edge length. The following is proved in [Luo07].

Lemma 4.3. If $L$ is an edge of a tetrahedron $s$ with an angle system and $D_{1}, D_{2}$ are two faces of $s$ having $L$ as an edge, then the length of $L$ in $D_{1}$ is the same as that in $D_{2}$.

Thus the following is well defined.

Definition 4.4. Let $\alpha=\left(\alpha_{12}, \cdots, \alpha_{34}\right) \in A S(s)$. The edge lengths of $\alpha$ are the numbers $\left(l_{i j}\right)_{i \neq j}$ defined as follows: $l_{i j}$ is the length of the edge $i j$ in the two faces of $T$ adjacent to the vertices $i$ and $j$.

4.4. A classification of simplices. It is now possible to describe a classification of 3-dimensional angled simplices. It is slightly more elaborate than the corresponding classification for Möbius triangles, because simplices can be "flipped" in two ways, depending on whether one or two vertices are in one of the copies of $H^{3}$ in $\tilde{H} S^{3}$. Here is the definition of the flip at the $i$-th vertex of $\alpha \in A S(s)$. See [Luo07] for more details. The $i$-th flipped simplex $\alpha^{\prime}=\left(\alpha_{12}^{\prime}, \ldots, \alpha_{34}^{\prime}\right)$ has angles $\alpha_{i j}^{\prime}=\alpha_{i j}$ for $j \neq i$ and $\alpha_{j k}^{\prime}=\pi-\alpha_{j k}$ for $j, k \neq i$. Geometrically, if $v_{1}, v_{2}, v_{3}, v_{4}$ are the vertices of a spherical simplex, then the flipped simplex (about the first vertex $v_{1}$ ) is the spherical simplex with vertices $-v_{1}, v_{2}, v_{3}, v_{4}$ where $-v_{1}$ is the antipodal point of $v_{1}$.

Lemma 4.5. Let $\alpha \in A S(s)$. After a permutation of $\{1,2,3,4\}$, $\alpha$ is of exactly one of the following types:

(1) spherical: all faces of $T$ are spherical triangles, and all edge lengths are in $i(0, \pi)$.

(2) hyperbolic: all faces of $T$ are hyperbolic triangles, and all edge lengths are in $\mathbb{R}_{<0}$.

(3) flipped hyperbolic: the face (234) is a hyperbolic triangle, while the faces adjacent to the vertex 1 are flipped hyperbolic triangles. The lengths of the edges $(12),(13),(14)$ are in $i \pi+\mathbb{R}_{>0}$, while the length of the other edges are in $\mathbb{R}_{<0}$.

(4) doubly flipped hyperbolic: all faces of $T$ are flipped hyperbolic triangles, the lengths of the edges (12) and (34) are negative numbers, while the length of the other edges are in $i \pi+\mathbb{R}_{>0}$.

(5) Euclidean: all faces of $T$ are Euclidean triangles; all edge lengths are zero.

(6) flipped Euclidean: the lengths of the edges (12), (13), 14) are equal to $i \pi$, while the lengths of $(14),(24),(34)$ are zero.

(7) doubly flipped Euclidean: the lengths of (12),(34) are zero, while all other edges have length $i \pi$. 
The terminology is based, as for triangles, on the idea of "flipping" a hyperbolic simplex: this means replacing one of its vertices, say $v_{1}$, by its antipode in $\tilde{H S} S^{3}$. The dihedral angles at all three edges not adjacent to $v_{1}$ are then replaced by their complement to $\pi$, and the effect on the edge lengths is as described in Lemma 4.5. Doubly flipping a hyperideal simplex means replacing two vertices by their antipodes in $\tilde{H} S^{3}$.

Proof of Lemma 4.5. Let $\alpha \in A S(s)$ and let $v_{1}, v_{2}, v_{3}, v_{4}$ be the vertices of $s$. We consider different cases, depending on the lengths of the edges of $s$ and in particular of its face $\left(v_{1}, v_{2}, v_{3}\right)$.

(1) $\left(v_{1}, v_{2}, v_{3}\right)$ is a spherical triangle; i.e., its edge lengths are in $i(0, \pi)$. Lemma 3.4, applied to the three other faces of $s$, shows that the lengths of the three other edges of $s$ are also in $i(0, \pi)$, it follows that $s$ is spherical.

(2) $\left(v_{1}, v_{2}, v_{3}\right)$ is hyperbolic. Then its edge lengths are negative, and considering Lemma 3.4 shows that there are only two possibilities.

(a) $\left(v_{2}, v_{3}, v_{4}\right)$ is hyperbolic, that is, its edge lengths are negative. Then, again by Lemma 3.4. the length of the edge $\left(v_{1}, v_{4}\right)$ is also negative, so that $s$ is hyperbolic.

(b) $\left(v_{2}, v_{3}, v_{4}\right)$ is flipped hyperbolic, that is, the lengths of the edges $\left(v_{2}, v_{4}\right)$ and $\left(v_{3}, v_{4}\right)$ are in $i \pi+\mathbb{R}_{>0}$. Then the length of $\left(v_{1}, v_{4}\right)$ is also in $i \pi+\mathbb{R}_{>0}$, so that $s$ is flipped hyperbolic.

(3) $\left(v_{1}, v_{2}, v_{3}\right)$ is flipped hyperbolic. We can suppose without loss of generality that the length of $\left(v_{1}, v_{2}\right)$ is in $\mathbb{R}_{<0}$ and the lengths of the two other edges are in $i \pi+\mathbb{R}_{>0}$. Two cases are possible.

(a) $\left(v_{1}, v_{2}, v_{4}\right)$ is hyperbolic; it then follows from Lemma 3.4 that $s$ is flipped hyperbolic.

(b) $\left(v_{1}, v_{2}, v_{4}\right)$ is flipped hyperbolic; it then follows from Lemma 3.4 that $s$ is doubly flipped hyperbolic.

(4) $\left(v_{1}, v_{2}, v_{3}\right)$ is Euclidean, so that all its edges have zero length. Lemma 3.4 then shows that there are two possible cases.

(a) $\left(v_{1}, v_{2}, v_{4}\right)$ is Euclidean. Then all edges of $s$ have zero length, and $s$ is Euclidean.

(b) $\left(v_{1}, v_{2}, v_{4}\right)$ is flipped Euclidean, so that $\left(v_{1}, v_{4}\right)$ and $\left(v_{2}, v_{4}\right)$ have length $i \pi$. In this case $s$ is flipped Euclidean.

(5) $\left(v_{1}, v_{2}, v_{3}\right)$ is flipped Euclidean. We can suppose without loss of generality that $\left(v_{1}, v_{2}\right)$ has zero length, while the lengths of the other two edges are equal to $i \pi$. There are again two cases to consider.

(a) $\left(v_{1}, v_{2}, v_{4}\right)$ is Euclidean, so that all its edge lengths are zero, and it easily follows that $s$ is flipped Euclidean.

(b) $\left(v_{1}, v_{2}, v_{4}\right)$ is flipped Euclidean, so that the edges $\left(v_{1}, v_{4}\right)$ and $\left(v_{2}, v_{4}\right)$ have length $i \pi$. Then $s$ is doubly flipped Euclidean.

According to the lemma, there are three types of angled simplices: Euclidean, hyperbolic and spherical. An angled simplex is of Euclidean (or hyperbolic) type if it can be flipped to a Euclidean (or hyperbolic) simplex. A spherical-type simplex is the same as a spherical simplex. The type of a simplex can be determined by the length of any of its edges. 
Corollary 4.6. Suppose $e$ is an edge of an angled simplex of length $l(e)$. Then $s$ is of

(1) Euclidean type if and only if $l(e) \in\{0, i \pi\}$,

(2) hyperbolic type if and only if $l(e) \in \mathbb{R}_{<0} \cup\left\{i \pi+r \mid r \in \mathbb{R}_{>0}\right\}$,

(3) spherical type if and only if $l(e) \in i(0, \pi)$.

In particular, if $e$ and $e^{\prime}$ are two edges of an angled simplex $s$, then their lengths $l(e)$ and $l\left(e^{\prime}\right)$ are in the same subset listed above.

4.5. Combinatorics of the space of simplices. The classification given in $\S 4.4$ can be interpreted in terms of the HS-geometry as follows, as for Möbius triangles in Lemma 3.7. Let $s$ be a simplex, and let $\alpha \in A S(s)$.

(1) If $\alpha$ is spherical, the $\alpha_{i j}$ are the dihedral angles of a unique simplex in $S^{3}$.

(2) If $\alpha$ is hyperbolic, the $\alpha_{i j}$ are the dihedral angles of a unique simplex in $H^{3}$.

(3) If $\alpha$ is flipped hyperbolic, the $\alpha_{i j}$ are the dihedral angles of a unique simplex in $\tilde{H S}{ }^{3}$, with three vertices in one of the copies of $H^{3}$ and one in the other.

(4) If $\alpha$ is doubly flipped hyperbolic, the $\alpha_{i j}$ are the dihedral angles of a unique simplex in $\tilde{H} S^{3}$, with two vertices in one of the copies of $H^{3}$ and two in the other.

(5) If $\alpha$ is Euclidean, the $\alpha_{i j}$ are the dihedral angles of a Euclidean simplex, unique up to homothety. They are also limits of sequences of angles of hyperbolic simplices.

(6) If $\alpha$ is flipped Euclidean, it is the limit of a sequence of angles of flipped hyperbolic polyhedra.

(7) Similarly, if $\alpha$ is doubly flipped Euclidean, it is the limit of a sequence of angles of doubly flipped hyperbolic polyhedra.

Consider now $A S(s)$ as the space of 6-tuples of angles in $(0, \pi)$ satisfying some linear inequalities. It contains some subdomains corresponding to the different types of simplices. It is interesting to consider the combinatorics of this decomposition of $A S(s)$. The definitions show clearly that any continuous path going from a simplex of spherical type to a simplex of hyperbolic type has to go through a simplex of Euclidean type. Moreover, the only way to go from a hyperbolic simplex to a doubly flipped hyperbolic simplex is through spherical simplices, and similarly for doubly flipped hyperbolic simplices.

\section{The Generalized VOlume}

5.1. The Schläfli formula. The last part of the picture considered here is the generalized volume, which is defined for the simplices in the extended hyperbolic space. There are several ways to define it. We use here the Schläfli formula, which we first recall for "usual" (spherical or hyperbolic) simplices. We refer to Mil94, Vin93, for a proof.

Lemma 5.1. For any 1-parameter family of spherical (resp. hyperbolic) simplices, its volume $V$ satisfies $2 d V=\sum_{e} \operatorname{Im}\left(l_{e}\right) d \alpha_{e}$ (resp. $\left.2 d V=\sum_{e} l_{e} d \alpha_{e}\right)$.

Note that the lengths considered here are those defined above, so that they are in $(0, i \pi)$ for spherical simplices and in $\mathbb{R}_{<0}$ for hyperbolic simplices. 
5.2. The generalized volume. The previous lemma leads to a fairly natural definition of a real-valued volume over the space of angled simplices.

Definition 5.2. Let $s$ be a tetrahedron and let $\omega$ be the 1-form (Schläfli 1-form) defined on $A S(s)$ by $2 \omega=\sum_{e}\left(\operatorname{Re}\left(l_{e}\right)+\operatorname{Im}\left(l_{e}\right)\right) d \alpha_{e}$.

Note that the Schläfli 1-form is a continuous 1-form defined on the 6-dimensional convex polytope $A S(s)$.

It is proved in [Luo07] that

Lemma 5.3. $\omega$ is closed.

Note that $\omega$ vanishes on the subspace of Euclidean simplices.

Definition 5.4. The generalized volume $V: A S(s) \rightarrow \mathbb{R}$ is the primitive of $\omega$ which vanishes on the Euclidean simplices.

There is another possibility, namely to define the volume as a complex-valued function, defining $\omega$ as $(1 / 2) \sum_{e} l_{e} d \theta_{e}$. The definition chosen here serves well for our purposes.

Note that $V$ corresponds to the usual volume on spherical and hyperbolic simplices by Lemma 5.1. The volume of Euclidean simplices is zero by definition. However, the volume of flipped and doubly flipped Euclidean simplices is not zero.

Lemma 5.5. Let $\alpha \in A S(s)$.

(1) Suppose that $\alpha$ is flipped Euclidean, with the lengths of the edges adjacent to $v_{1}$ equal to $i \pi$ and the other lengths equal to 0 . Then

$$
V(\alpha)=\pi\left(\alpha_{12}+\alpha_{13}+\alpha_{14}-\pi\right) .
$$

(2) Suppose that $\alpha$ is doubly flipped Euclidean, with $l_{12}=l_{34}=0$ and the other lengths equal to $i \pi$. Then

$$
V(\alpha)=\pi\left(\alpha_{13}+\alpha_{14}+\alpha_{23}+\alpha_{24}-2 \pi\right) .
$$

Note that in each case the volume, without the factor $\pi$, is equal to the area of a spherical polygon - this will be useful below.

Proof of Lemma 5.5. For the first case, consider a small deformation that increases slightly the $\alpha_{1 i}, 2 \leq i \leq 4$. This deforms $\alpha$ into a spherical simplex $\alpha^{\prime}$, with vertices $v_{2}, v_{3}$ and $v_{4}$ very close to the antipode of $v_{1}$. The (spherical) Schläfli formula, applied to a 1-parameter formula deforming this simplex to a segment of length $\pi$, shows that the volume of this simplex is equal to $\pi\left(\alpha_{12}^{\prime}+\alpha_{13}^{\prime}+\alpha_{14}^{\prime}-\pi\right)$, and the result follows for $\alpha$.

The same argument works in the second case; the corresponding spherical simplex now has $v_{1}, v_{2}$ very close and almost antipodal to both $v_{3}$ and $v_{4}$.

There is a quite different way to define this "volume" of domains in the extended hyperbolic space, in terms of an analytic continuation [CK06].

5.3. Smoothness. For a closed triangulated 3-manifold $(M, T)$, the volume $V$ of an angle system $x \in A S(T)$ is the sum of the volume of its angled 3-simplices. Thus $v: A S(T) \rightarrow \mathbb{R}$ is a $C^{1}$ smooth function. Moreover it is real analytic outside the set of Euclidean-type simplices. 


\section{CRitical points}

This section contains the proof of Theorem 1.3 .

6.1. Gluing conditions. Suppose $(M, T)$ is a connected triangulated closed 3manifold so that $A S(T) \neq \emptyset$. We will consider the volume optimization $V$ : $A S(T) \rightarrow \mathbb{R}$.

Lemma 6.1. Let $\theta_{0} \in A S(T)$ be a critical point of $V$ on $A S(T)$. Then, for each edge $e$ of $T$, the lengths of e for all the simplices containing it are equal.

This follows from the definition of $V$ and from Lemma 5.1. By the classification lemma, Lemma 4.5, and the connectivity of $M$, we see that all simplices in $\theta_{0}$ have the same spherical or hyperbolic or Euclidean type.

If $\theta$ is a local maximum point of $V$, then it cannot happen that all 3 -simplices in $\theta$ are Euclidean simplices. Indeed, if otherwise, the volume of $\theta$ is zero. However, if we perturb $\theta$ slightly in $A S(T)$ to obtain a new point $\theta^{\prime}$, then all simplices in $\theta^{\prime}$ can be hyperbolic and spherical simplices. Thus $V\left(\theta^{\prime}\right)>V(\theta)$, which contradicts the local maximum condition.

According to Lemma 3.6, for non-Euclidean-type simplices, edge lengths determine the isometry type. So we obtain

Corollary 6.2. Suppose $\theta$ is not of Euclidean type. The faces of the simplices can be glued isometrically. Furthermore, $\theta$ defines in this way either a spherical structure or an HS-structure $h$ on $M$.

Indeed, there are two possibilities. Namely either all simplices in $\theta$ are of spherical type or they are all of hyperbolic type. In the spherical type case, all simplices are spherical and are glued by isometries so that the sum of angles around each edge is $2 \pi$. Thus we obtain a spherical metric on $M$. In the case where all simplices are of hyperbolic type, by Lemma 4.5, we realize each simplex in $\theta$ as a geometric tetrahedron in $\tilde{H} S^{3}$ so that their faces can be glued isometrically. Thus, we obtain an HS-structure on $M$. Indeed, there are two subcases which could occur. In the first case, all simplices are hyperbolic. Thus we obtain a hyperbolic metric on $M$. In the second case, some simplex is flipped hyperbolic. Then we obtain an HS-structure on $M$ by gluing these geometric tetrahedra in HS-geometry.

Note that all vertices of $T$ are in the hyperbolic part of this HS-structure.

6.2. Normal spheres in HS-structures. Continuing the proof of Theorem 1.3. we consider here an HS-structure $h$ on $M$, along with a triangulation $T$ with all vertices of $T$ in the hyperbolic part of $h$. Suppose moreover that the de Sitter part $M_{d S}$ for $h$ is non-empty. Let $M_{0}$ be a connected component of $M_{d S}$.

Then $M_{0}$ is geodesically complete, so it is isometric either to the de Sitter space $d S^{3}$ or to its quotient by the antipodal map; indeed it is well known that the only complete 3-dimensional de Sitter manifolds are $d S^{3}$ itself and its quotient by the antipodal map (it is proved in Wol84, §11.1] that such manifolds have finite fundamental group, and an elementary argument using the dual action on $H^{3}$ then shows that this fundamental group is either trivial or $\mathbb{Z} / 2 \mathbb{Z}$ ). Recall that the de Sitter space has constant curvature 1 . Therefore any space-like plane in the tangent space to $M_{0}$ at a point is tangent to a (unique) totally geodesic space-like plane in $M_{0}$, which is homeomorphic either to $S^{2}$ (in the first case) or to $\mathbb{R} P^{2}$ (in the 
second case). Each of those totally geodesic surfaces is a normal surface in the triangulation $T$ of $M$.

This simple argument shows that each connected component of the de Sitter part of $h$ corresponds to a normal surface in $(M, T)$.

6.3. Normal spheres for Euclidean critical points of $V$. In this section we consider the same question as in 46.2 , about normal surfaces in $(M, T)$, but for critical points of $V$ for which all simplices are of Euclidean type. The arguments are somehow similar but are less geometric and more combinatorial, because the geometric structures on the simplices cannot be glued to obtain a geometric structure of Euclidean type on $M$.

We have seen in $\$ 5.2$ that to each flipped (resp. doubly flipped) Euclidean simplex $s$ in $T$ can be associated a spherical triangle (resp. a quadrilateral). The edges of this triangle (resp. quadrilateral) are associated to the 2-faces of $T$ which have exactly two edges of length $i \pi$. Each such face bounds two simplices which are both either flipped or doubly flipped. It follows that the triangles (resp. quadrilaterals) can be glued along their edges to obtain a closed surface $\Sigma$ (which in general is not connected); however this gluing cannot in general be isometric for the spherical metrics since the lengths of the edges do not match. Moreover, the vertices of the triangulation of $\Sigma$ correspond to the edges of $T$ of length $i \pi$.

Remark 6.3. The angles of the triangles (resp. quadrilateral) at each vertex sum up to $2 \pi$.

Proof. The angles of the triangles (resp. quadrilateral) adjacent to each vertex of $\Sigma$ are equal to the angles of the simplices of $T$ at the corresponding edge of length $i \pi$. Those angles sum up to $2 \pi$ by definition of an angle structure on $T$.

Corollary 6.4. Each connected component of $\Sigma$ is homeomorphic either to the sphere or to the projective plane. The sum of the areas of the faces of $\Sigma$ is an integer multiple of $2 \pi$.

Proof. Let $\Sigma_{0}$ be a connected component of $\Sigma$, let $F_{0}$ be the set of its 2-faces, and let $V_{0}$ be the set of its vertices. Given $f \in F_{0}$ and $v \in V_{0}$, we write $v \simeq f$ if $v$ is adjacent to $f$; in this case we call $\theta_{f, v}$ the angle of $f$ at $v$.

Let $a(f)$ be the area of the face $f$ of $\Sigma$. For each face $f \in F_{0}$ of $\Sigma_{0}$, we have by the Gauss-Bonnet formula

$$
\sum_{v \in V_{0}, v \simeq f}\left(\pi-\theta_{f, v}\right)=2 \pi-a(f) .
$$

Summing over the faces of $\Sigma_{0}$ yields that

$$
\sum_{f \in F_{0}}\left(\sum_{v \in V_{0}, v \simeq f}\left(\pi-\theta_{f, v}\right)\right)=2 \pi \# F_{0}-\sum_{f \in F_{0}} a(f) .
$$

The number of wedges in the triangulation of $\Sigma_{0}$ is twice the number of edges, which we denote by $\# E_{0}$. Therefore

$$
\sum_{f \in F_{0}} \sum_{v \in V_{0}, v \simeq f} \pi=2 \pi \# E_{0} .
$$


Moreover the angles of the faces at each vertex sum up to $2 \pi$, so that

$$
\sum_{f \in F_{0}} \sum_{v \in V_{0}, v \simeq f} \theta_{f, v}=2 \pi \# V_{0} .
$$

Using the definition of the Euler characteristic, we obtain that

$$
\sum_{f \in F_{0}} a(v)=2 \pi \# V_{0}-2 \pi E_{0}=2 \pi F_{0}=2 \pi \chi\left(\Sigma_{0}\right)
$$

and both parts of the corollary follow immediately.

Corollary 6.5. At a critical point of $V$ where all simplices are of Euclidean type, $V$ is an integer multiple of $2 \pi^{2}$.

Proof. Lemma 5.5 shows that the volume of each flipped (resp. doubly flipped) simplex is equal to $\pi$ times the area of the corresponding triangle (resp. quadrilateral) in $\Sigma$. So the total volume is $\pi$ times the area of $\Sigma$, so that it is a non-negative integer multiple of $2 \pi^{2}$.

The proof of Theorem 1.3 is obtained by putting together the results of this section.

\section{Further Questions}

The main point presented here is that extended hyperbolic structures have a natural role when constructing geometric structures on manifolds by maximization of volume over triangulated manifolds. This leads to a number of questions, for which answers would presumably help make progress on the understanding of geometric structures on 3-manifolds.

Question 7.1. If $M$ is a connected sum of several hyperbolic 3-manifolds, does $M$ support an HS-structure?

Another, more general, question is whether the constructions considered here can be extended to encompass angle structures with some ideal vertices. This would mean allowing angle structures on simplices for which the sum of the angles at a vertex is equal to, rather than greater than, $\pi$. Our hope is that such ideal vertices would permit critical points of the volume to realize torus decompositions of toroidal 3-manifolds. Another possibility, adding some flexibility to the construction, would be to allow for vertices in the de Sitter part of the extended hyperbolic space.

Another natural question is of course to understand the critical points of $V$ on the boundary of $A S(T)$, hopefully showing that those boundary critical points correspond to collapsings.

A last, more technical question, is whether existence of a critical point of $V$ on $A S(T)$ for which all simplices are of Euclidean type has topological consequences on $M$. For instance, if all simplices are Euclidean (rather than only of Euclidean type), does it follow that $M$ admits a Euclidean metric or more generally that $M$ is a connected sum of Seifert fibered spaces? This is not obvious since the angles of the simplices add up to $2 \pi$ at the edges of $T$, but the edge lengths do not match so that the faces of the simplices cannot be isometrically glued. 


\section{ACKNOWLEDGEMENT}

The authors are grateful to an anonymous referee for many relevant remarks which led to clear improvements of the content of the paper.

\section{REFERENCES}

[CK06] Yunhi Cho and Hyuk Kim. Volume of $C^{1, \alpha}$-boundary domain in extended hyperbolic space. J. Korean Math. Soc., 43(6):1143-1158, 2006. MR2264675 (2007j:51014)

[KL] A. Kitaev and Feng Luo. Existence of angle structures on 3-manifolds, in preparation.

[Luo93] Feng Luo. Triangulations in Möbius geometry. Trans. Amer. Math. Soc., 337(1):181-193, 1993. MR 1123456 (93g:57026)

[Luo07] Feng Luo. Volume and angle structures on 3-manifolds. Asian J. Math., 11(4):555-566, 2007. MR:2402938 (2009b:57038)

[Mil94] John Milnor. The Schläfli differential equality. In Collected papers, vol. 1. Publish or Perish, 1994. MR1277810 (95c:01043)

[Sch98] Jean-Marc Schlenker. Métriques sur les polyèdres hyperboliques convexes. J. Differential Geom., 48(2):323-405, 1998. MR1630178 (2000a:52018)

[Sch01] Jean-Marc Schlenker. Convex polyhedra in Lorentzian space-forms. Asian J. Math., 5(2):327-363, 2001. MR1868937 (2003f:53127)

[Vin93] E.B. Vinberg, editor. Geometry II, Geometry of Spaces of Constant Curvature, volume 29 of Encyclopaedia of Mathematical Sciences. Springer, 1993. MR1254931 (94f:53002)

[Wol84] Joseph A. Wolf. Spaces of constant curvature. Publish or Perish Inc., Houston, TX, fifth edition, 1984. MR.928600(88k:53002)

Department of Mathematics, Rutgers University, Piscataway, New Jersey 08854

Institut de Mathématiques de Toulouse, UMR CNRS 5219, Université Toulouse III, 31062 Toulouse Cedex 9, France 